\title{
A Study of the Optical Properties of Cosmetics Measured by Polarized Light Goniophotometry
}

\author{
Sun Kyung Choi*, Young Jun Yang, Kyung Nam Kim, Jaewook Choi, \\ Yeong Jin Choi, and Sang Hoon Han \\ Amorepacific Corporation R\&D Center, 314-1 Bora-dong, Giheung-gu, Yongin 446-729, Korea
}

(Received October 21, 2011 : revised November 24, 2011 : accepted December 16, 2011)

\begin{abstract}
Polymethylmethacrylate powders are commonly used to improve the spreadability and blurring effects of powder foundation cosmetics. In this study, the opacity and haze effect of four types of polymethylmethacrylate powders and their $10 \mathrm{wt} \%$ powder foundation formulation was evaluated. The distorted random hemispherical particulates with both concave and convex portions showed the highest haze and opacity value as a single powder substance. And as a formulated phase, the powder foundation formulation containing particulates with fine pores provided the highest haze and opacity value. We also examined the quantity of reflected light over the angular range $20-75^{\circ}$ by polarized light goniophotometry. The total reflected light intensity, surface-reflected light, and internally reflected light were characterized. These studies demonstrated that hemispherical PMMA powders produce natural brilliance by creating high internally reflected light which is similar to the total reflection profile of artificial skin.
\end{abstract}

Keywords : Cosmetics, Polarized light, Goniophotometry, Reflection

OCIS codes : (120.2440) Filters; (150.3045) Industrial optical metrology; (230.5160) Photodetectors;

(240.6645) Surface differential reflectance; (290.5850) Scattering, particles

\section{INTRODUCTION}

The spreading of light across an infant's skin surface and the layer inside, produces a soft even luminosity and translucency. The 'soft-focus' luminosity of skin decreases over time due to the accumulation of photodamage. Also, contrast increases as topographic features such as lines, furrows, and wrinkles increase as the skin ages $[1,2]$. In addition to age, a person's genetics also affect skin light reflectance patterns and skin translucency [3, 4]. Numerous studied have been performed to visualize the normal and diseased skin morphologies to measure the thickness using optical sensors $[5,6]$. Skin care products improve skin by treating the skin over time, and makeup products provide immediate physical changes in the light reflection properties by covering the spots and lines. Today, the study of skin colors and textures is applicable not only to the field of dermatology and cosmetics, but also in the field of computer graphics [7-9].

The principal concern of makeup products in the 80 's through the 90 's was coverage. However, the makeup trend in the 2000s has transferred from high coverage to facilitating natural skin expression. For this aspect, scattering effects, which dispersed light to create a soft-focus effect, received significant attention. Light diffusion patterns are primarily determined by particulate powders, which are used in pressed powder and loose powder cosmetics, and the properties of such powders have been widely studied.

The trend of focusing on natural skin expression is expected to hold out for a while yet. From this perspective, the development of cosmetic materials has focused on formulation and material properties. Formulation studies have focused on decreasing the use of titanium dioxide, which has a high refractive index and is used as spherical particulates that produce high blurring effects. Material studies have focused on hybrid technology. The hybrid of transparent powder and high refractive index pigment makes the skin coverage natural. For example, a hybrid material containing low-refractive index materials such as mica or boron nitride, along with the high-refractive index material titanium dioxide, gives human skin a natural-looking expression and evenly distributes the titanium dioxide across the surface. As another example,

\footnotetext{
*Corresponding author: csk@amorepacific.com

Color versions of one or more of the figures in this paper are available online.
} 
when high blurring polymethylmethacrylate (PMMA) or silica is treated with titanium dioxide, the result is human skin with a non-artificial appearance through facilitating an even distribution and improving light diffusion in all directions [10].

In this study, we measured the skin appearance effects of cosmetics containing powdered pigments and the powder particle itself by using a colorimeter and polarized light goniophotometry measurements with a halogen polarized lamp. We attempted to spread the light uniformly by controlling the formulation within the stability boundary of cosmetics.

\section{METHODOLOGY}

The goal of this study was to analyze the reflectance and transmission patterns of light after makeup is applied. We performed colorimetry measurements using a xenon lamp and polarized light goniophotometry measurements using a halogen lamp. Colorimetry measurements (using a HunterLab, model Colorquest XE) were conducted using $\mathrm{D}_{65}$ daylight illumination. Haze and opacity effects were measured in both powder particles form and powder foundation formulation form. The internal reflected light, surface-reflected light, and total reflected light were quantified as a function of the angle of incident light.

\subsection{Opacity}

The opacity of the powder particle forms and their formulations were determined according to the Hunterlab method in the reflectance mode. The opacity (Y) of the samples was calculated as the relationship among the opacity of each sample on the black standard ( $\mathrm{Y}_{\text {black backing }}$ ) and the opacity of each sample on the white standard ( $\mathrm{Y}_{\text {white backing). This }}$ calculation $\left(\mathrm{Y}=\mathrm{Y}_{\mathrm{b}} / \mathrm{Y}_{\mathrm{w}}\right)$ was made automatically by the Universal Software (Hunterlab Associates Laboratory).

$$
\text { Opacity }(\mathrm{Y})=\frac{\mathrm{Y}_{\text {black backing }}}{\mathrm{Y}_{\text {white backing }}} \times 100
$$

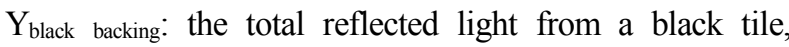
measured at the reflection port

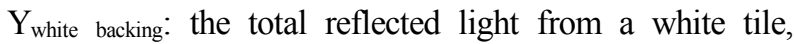
measured at the reflection port
}

A high opacity indicates that the quantity of light reflected from a black tile was similar to that reflected from a white tile. And a low opacity indicates that the reflected light is sensitive to the tiles' properties [11].

\subsection{Haze}

The haze of the powder particle forms and their formulations were determined according to the Hunterlab method in the transmission mode. Haze measurements were obtained by the ratio of quantity of diffuse light ( $\left.\mathrm{Y}_{\text {diffuse transmission }}\right)$ to the total light ( $\left.\mathrm{Y}_{\text {total transmission }}\right)$ transmitted by a specimen. This calculation $\left(\right.$ Haze $\left.=Y_{d} / Y_{t}\right)$ was made automatically by the Universal Software (Hunterlab Associates Laboratory) also.

$$
\text { Haze }=\frac{Y_{\text {diffuse transmission }}}{Y_{\text {total transmission }}} \times 100
$$

$Y_{\text {diffuse transmission: Diffuse component of the transmitted light }}$ $Y_{\text {total transmission: Total transmitted light }}$

The haze effect combines with the blurring effect to distribute light throughout a cosmetic applied to the skin.

\subsection{Polarization Goniometry}

The surface-reflected light and internal reflection of powder foundation formulation were measured by goniophotometer. The setup of the polarized goniophotometer system with a halogen lamp is shown in Fig. 1.

Both input and detected light are polarized. The incident light angle is $45^{\circ}$ and a convergent angle ranges over $20-75^{\circ}$, with a moving detector. The detection polarizer can be rotated to collect either parallel or perpendicular polarized light [12-16]. Each quantity of light can be calculated from the quantity of parallel filtered and vertically filtered light.

The quantity of internally reflected light:

$$
\mathrm{I}_{\text {internally reflected light }}=2 \times \mathrm{I}_{\text {vertical }}
$$

The quantity of surface-reflected light:

$$
\mathrm{I}_{\text {surface-reflected light }}=\mathrm{I}_{\text {parallel }}-\mathrm{I}_{\text {vertical }}
$$

The quantity of totally reflected light:

$$
\mathrm{I}_{\text {total }}=\mathrm{I}_{\text {internally }}+\mathrm{I}_{\text {surface }}=\mathrm{I}_{\text {parallel }}+\mathrm{I}_{\text {vertical }}
$$

$\mathrm{I}_{\text {crossed: the quantity of light passing through the crossed }}$ polarized filters

$\mathrm{I}_{\text {parallel }}$ : the quantity of light passing through the parallel polarized filters [17]

\subsection{Sample Preparation}

We measured the haze and opacity of four types of PMMA powders and their powder foundation formulations containing $10 \mathrm{wt} \%$ powder particulates. The powders were

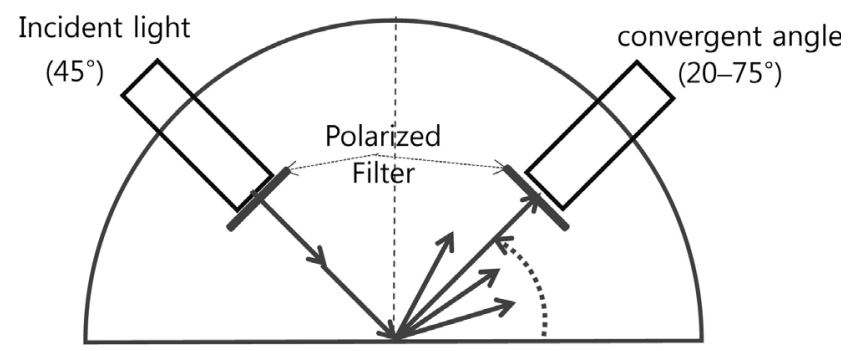

FIG. 1. Schematic diagram of the polarized light goniophotometer. 
denoted as follows: A indicates Artpearl 7P (Negami Industry), which contains spherical particulates with smooth surfaces; B indicates Maki bead 150 (Daito Kasei), which contains particulates with fine pores that facilitate oil absorption; $\mathrm{C}$ indicates SunPMMA-X (Sunjin chemical), which contains distorted random hemispherical particulates with both concave and convex portions; and D indicates 3D-PW (Daito Kasei), which contains hemispherical PMMA. For the powder form test, we applied the powder particles over a $3 \mathrm{M}$ tape on a slide glass, and spread it over a surface using a cosmetic puff. The weights of the powders were $0.01 \mathrm{~g}$. For the formulation test, we considered the customer's method for applying the samples. A cosmetic puff which was drawn 3 times over the powder pact was set up to the Sun Rheometer, and was spread 4 times automatically with pressing by $200 \mathrm{~g}$ weights. A $3 \mathrm{M}$ tape was used to measure the opacity and haze effect sample, and a Bio Skin artificial skin plate (Beautylax Co. Ltd.) was used to measure the internal reflections and surface reflections of light as a function of the convergent angle using a goniophotometer.

\section{EXPERIMENTAL RESULTS}

\subsection{The Opacity and Haze Effects of the Various Shape of Polymethylmethacrylate Particulate Powder}

The opacity and haze effects of a highly blurring and highly spreadable PMMA were measured. Four types of PMMA powder were selected for this measurement by their shape. The characteristic of powders are shown in Table 1. For measurement of oil absorption, caprylic capric triglyceride was used.

The opacity and haze were measured both for powder form and for formulations containing $10 \mathrm{wt} \%$ of the material. The results are discussed in Section 1.1 for powder form, and for powder foundation form in Section 1.2.

TABLE 1. Comparison of the oil absorbability and appearance of each powder

\begin{tabular}{c|c|c}
\hline \hline Material & $\begin{array}{c}\text { The quantity of } \\
\text { absorbed oil }\end{array}$ & Appearance \\
\hline $\mathrm{A}$ & 56.2 & \\
\hline $\mathrm{B}$ & 155.7 & \\
\hline $\mathrm{C}$ & 395.2 & \\
\hline $\mathrm{D}$ & 57.6 & \\
\hline
\end{tabular}

TABLE 2. Haze and opacity properties of each powder

\begin{tabular}{c|c|c}
\hline \hline Material & Haze & Opacity \\
\hline A & $93.63 \pm 0.96$ & $38.77 \pm 0.64$ \\
\hline B & $96.30 \pm 0.64$ & $50.91 \pm 0.57$ \\
\hline C & $98.27 \pm 0.73$ & $65.08 \pm 0.32$ \\
\hline D & $93.48 \pm 0.62$ & $56.38 \pm 0.94$ \\
\hline
\end{tabular}

\subsubsection{Opacity and Haze Properties of Each Material}

The opacity and haze properties were measured by colorimetry using $0.01 \mathrm{~g}$ samples of each material, spread as described. The reported measurements are the average of five independent measurements. Table 2. show the haze and opacity ability of each powder.

The haze and opacity ability was measured differently depending on the powder shape. The properties of particulates with surface pores, B, were compared with those with smooth surfaces, A. The pores of powder B gave higher oil absorbability and also roughened the surface which resulted in higher scattering effects than for A. The higher scattering effect increased both the opacity of the coverage effects and the haze effect. Powder $\mathrm{C}$ had the highest haze and opacity value. There are two reasons. First, is the effect of specific gravity. And second, is the effect of the uneven surface. Both because of the concave areas and convex areas. The concave area provides much higher oil absorbability giving much lower specific gravity than other powders. Because of this low specific gravity, the number of particles for the same weight should be higher. Secondly, the presence of concave and convex areas made powder C's applied surface uneven; it also helped the high scattering effects and provided high haze and opacity ability. From comparing $\mathrm{A}, \mathrm{B}$ and $\mathrm{C}$, we determined that the shape of particles is more important than the fine pores in the haze and opacity results and we predicted the degree of roughness of an applied surface by the oil absorbability. The sample D is also a hemispherical particle like $\mathrm{C}$, but has a flat side instead of a concave one. This flat side contacted the skin of the face, so the applied powder's surface was very similar to sample $\mathrm{A}$. This is the reason that $\mathrm{D}$ gave very similar haze value to $\mathrm{A}$. But since $\mathrm{D}$ is attached to the facial skin by face when A contacts it only as a spot, the applied layer of powder D is denser and resulted in high opacity properties.

\subsubsection{The Haze and Opacity Effect of the Spherical Powder in a Powder Foundation}

Each formulation was prepared with 10wt\% PMMA to characterize the effect of the opacity and haze. Differently from experimental method for the powder form, we prepared the samples using a Sun Rheometer according to the customer method for cosmetics as described. The haze and opacity ability of each formulation is shown in Table 3 .

The result was different from the result of the powder 
TABLE 3 . Haze and opacity properties of each formulation

\begin{tabular}{c|c|c}
\hline \hline Material & Haze & Opacity \\
\hline $\begin{array}{l}\text { Formulation 1 } \\
\text { (containing A) }\end{array}$ & $80.36 \pm 0.63$ & $28.23 \pm 0.38$ \\
\hline $\begin{array}{c}\text { Formulation 2 } \\
\text { (containing B) }\end{array}$ & $87.92 \pm 0.51$ & $38.97 \pm 0.81$ \\
\hline $\begin{array}{c}\text { Formulation 3 } \\
\text { (containing C) }\end{array}$ & $81.09 \pm 1.14$ & $27.76 \pm 0.59$ \\
\hline $\begin{array}{l}\text { Formulation 4 } \\
\text { (containing D) }\end{array}$ & $78.80 \pm 0.62$ & $27.12 \pm 0.42$ \\
\hline
\end{tabular}

form because of the effects of the other powder. Especially, the gap of opacity value decreased. The reason was that titanium dioxide commonly inserted in the formulation for coverage had a high refractive index that gave a largre influence to opacity than PMMA. The opacity and haze effect of formulation 2 was higher than for formulation 1 , because of the scattering of light by the pores over the surface. These results are similar to the results obtained from the powder form. But formulation 3's opacity and haze effect was not higher than that of formulaion 2. It is because powder $\mathrm{C}$ had a concave and a rough applied surface in powder condition, but the concavities were filled with other small sized particles in the formulation condition and the effects by concavities could not affect the results. And among the formulations, the formulation 4 gave the lowest opacity and haze value. We assumed that powder D has a smooth surface so the formulation 4 with the powder $\mathrm{D}$ behaved like formulation 1. Moreover the flat side located in powder foundation form lowered the diffusing ability, so the opacity and haze value is assumed to be the lowest in formulation 4 .

\subsection{Reflection of Convergent Light}

Light reflected from the human skin can be classified as surface-reflected light and internal reflection of transmitted light. These two types of reflected light can be measured using a light source and two polarized filters. Older skin tends to internally reflect smaller quantities of light; however, quantitative studies of the internally reflected and surfacereflected light remain unexplored. The samples prepared to measure the quantity of reflected light were spread using a rheometer and the light reflectance properties were measured using a polarized goniophotometer.

\subsubsection{Surface-reflected Convergent Light}

The quantity of surface-reflected light was measured through parallel polarized and vertically polarized filters. This measurement corresponds to the glossiness of skin. The graph in Fig. 2 plots the quantity of surface-reflected convergent light at each angle, calculated from the difference between the horizontal and vertical filters.

The quantity of surface light reflected by formulation 1

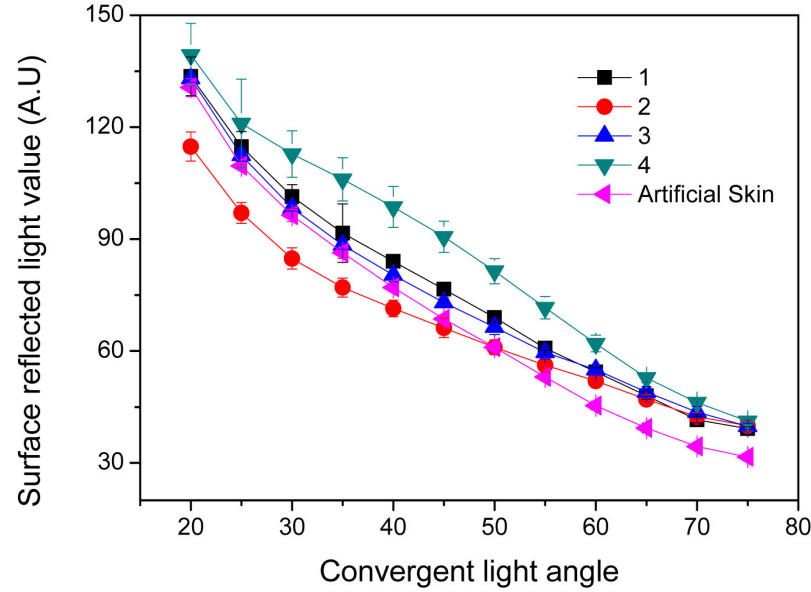

FIG. 2. Measurements of the surface-reflected convergent light.

and 3 shows higher intensities than that of the artificial skin through the convergent angle from $20^{\circ}$ to $75^{\circ}$. Tthe gap with artificial skin was particularly greater at over $45^{\circ}$. The quantity of surface light reflected by formulations 2 and 4 was significantly different from that of artificial skin. At first, the surface reflected light intensity of formulation 2 was lower and had a gentler slope than that of surface light reflected by the bare artificial skin. The pores of powder B enhanced the scattering effect and these properties provided a similar intensity over the angle from $20^{\circ}$ to $75^{\circ}$. On the other hand, the formulation containing hemispherical particulate PMMA showed the highest intensity of surfacereflected light. The flat regions of the hemispherical particulates contacted the skin during spreading of the powder foundation. The facts that hemispherical particles could attach on the skin effectively and that it made dense cosmetics layers were the reason that the light scattered on formulation 4 provided higher amounts of surface light than other formulations. From these results, we found that formulation 2 with porous powder gave a large soft-focus effect and matte texture for the face, and formulation 4 with hemispherical powder provided gloss over the face.

\subsubsection{Intemally Reflected Convergent Light}

The quantity of internally reflected light was measured through vertically polarized filters. Internally reflected light passes through the dermis or the epidermis of the skin and is scattered multiple times by collagen fibers. So the light path of the internally reflected light is longer and more extensively scattered compared to surface-reflected light.

The effect of the intensity of internally reflected light for each formulation spread onto artificial skin was smaller than the effect of the surface-reflected light for all angles of convergent light. Only the quantity of internally reflected light of formulation 2, containing B with pores, provided higher intensities than others. The pores of powder B on the outside of the cosmetic layer made the travel length of light longer 


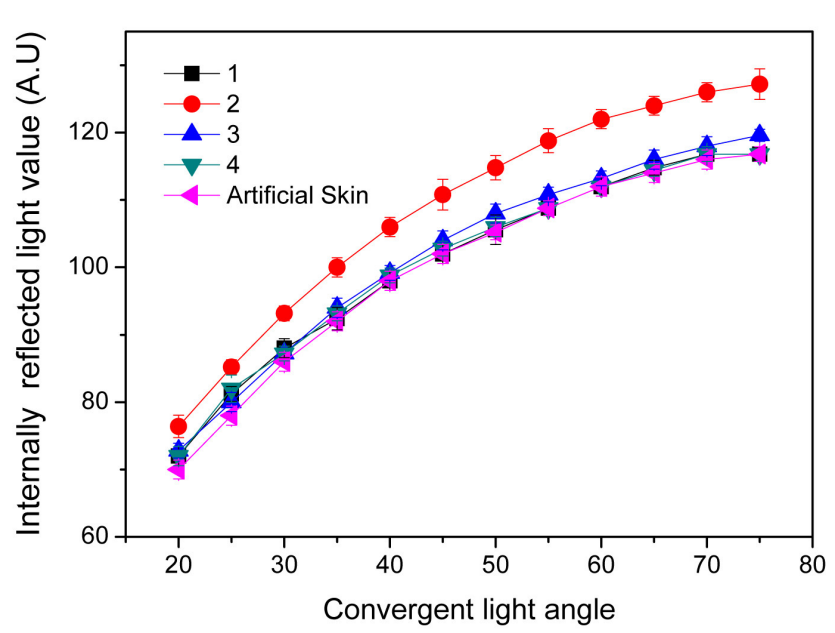

FIG. 3. Measurements of the internally reflected convergent light.

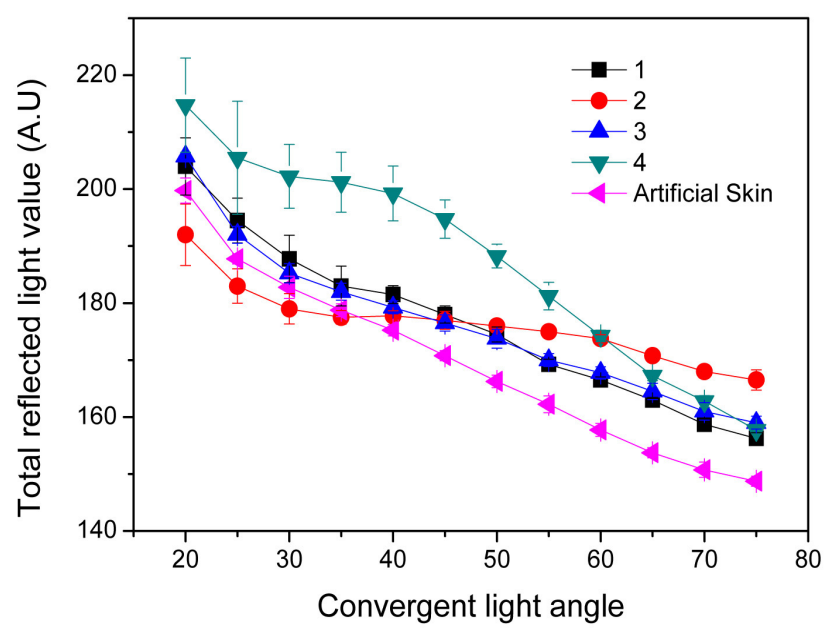

FIG. 4. Measurements of the total reflection of convergent light.

and gave effects similar to the light traveling inside the dermis or epidermis. From this result we found out that most light is reflected from the outside of cosmetic layer, not from the inside. So the approach to change the surface-reflected light rather than the internally reflected light is more effective in cosmetics.

\subsubsection{Total Reflection of Convergent Light}

The total reflection of light is calculated as the sum of the surface-reflected and internally reflected light. Figure 4 shows the total reflection of convergent light from the artificial skin on which the powder foundations containing 10wt\% PMMA has been spread.

The total intensity of reflected light was higher for formulations 1, 3, and 4 over the full angular range than for the sample without powder application. And the total quantity of reflected light decreased over the angle range $20-35^{\circ}$ and increased over the angle range $35-75^{\circ}$ for formulation 2 . The increase in the quantity of light indicates the effects of the cosmetics, and the higher quantity of the intensity, the cosmetic formulation produced a more gorgeous face. Formulation B, with surface pores, showed an overall low intensity, because of the low slope in the surface intensity of light. This gentle slope means that formulation 2 reflects the light evenly and could give downy effects after putting the make-up on. We also found that formulations 1 and 3 produced higher amount of light compared to artificial skin, so these formulations can give a bright expression. The most meaningful formulation for the best face expression was formulation 4 . Formulation 4 showed the highest intensity of reflection, and the pattern of light was also similar to artificial skin. This result indicates that formulation 4 makes the face brighter and also gives a natural complection.

\section{DISCUSSION AND CONCLUSIONS}

The trend in cosmetics has been shifted toward enhancing natural skin expression. We investigated the opacity and haze effect upon different shapes of PMMA powders used to achieve blurring effects in cosmetics. We also quantified the internally reflected, surface-reflected, and total reflected light as a function of the angle of convergent light. This study measured the light behavior on or in the human skin for a variety of particulate morphologies. These measurements and results provide guidelines for the formulation of cosmetic products. For a cosmetic product to achieve a brilliance effect, a high intensity of the total reflected light is needed. And for natural cosmetic expression, the patterns of total quantity of reflected light should be similar to that of bare artificial skin.

Formulation 2, containing fine pore PMMA particles, gave a soft-focus effect and downy face according to the even intensity over the convergent angles. But we concluded the formulation 4 to be the best. Formulation 4 which contains hemispherical PMMA particulates, amplified the brilliant effect of cosmetic products according to the strongest intensity of total reflected light, and also enabled a natural looking face by showing similar reflection patterns with the artificial skin.

This study provides objective experimental criteria for estimating the qualitative effects of a cosmetic product. This work can easily be extended to in vivo measurement of the optical properties of a cosmetic on human skin and also to subjective perception measurements.

\section{ACKNOWLEDGMENT}

This study was supported by a grant from the Korea Healthcare Technology R\&D Project, Ministry of Health \& Welfare, Republic of Korea (Grant No. A103017). 


\section{REFERENCES}

1. P. J. Matts, "New insight into skin appearance and measurement," Journal of Investigative Dermatology Symposium Proceedings 13, 6-9 (2008).

2. R. R. Anderson, "Polarized light examination and photography of skin," Arch. Dermatol. 127, 1000-1005 (1991).

3. Z. Jiang and P. K. Kaplan, "Point-spread imaging for measurement of skin translucency and scattering," Skin Research and Technology 14, 293-297 (2008).

4. T. Igarashi, K. Nishino, and S. K. Nayar, "The appearance of human skin," Technical Report CUCS-024-05, University of Columbia, New York, NY 10027, USA, June (2005).

5. S. H. Choi, W. H. Kim, Y. J. Lee, H. Lee, W. J. Lee, J. D. Yang, J. W. Shim, and J. W. Kim, "Visualization of epidermis and dermis cells in ex vivo human skin using the confocal and two-photon microscopy," J. Opt. Soc. Korea 15, 61-67 (2011).

6. D. S. Ho, E. H. Kim, I. D. Hwang, K. S. Shin, J. T. Oh, and B. M. Kim, "Optical skin-fat thickness measurement using miniaturized chip LEDs: a preliminary human study," J. Opt. Soc. Korea 13, 304-309 (2009).

7. N. Tsumura, N. Ojima, K. Sato, M. Shiraishi, H. Shimizu, H. Nabeshima, S. Akazaki, K. Hori, and Y. Miyake, "Imagebased skin color and texture analysis/synthesis by extracting hemoglobin and melanin information in the skin," SIGGRAPH, ACM, 770-779 (2003).

8. D. H. Kim and M. Y. Kim, "Skin color analysis in HSV color space and rendering with fine scale skin structure,"
Lecture Notes in Computer Science 4035, 254-264 (2006).

9. P. Harnrahan and W. Kruenger, "Reflection from layered surfaces due to subsurface scattering," SIGGRAPH, ACM, 187-194 (1993).

10. T. Okuura and Y. Isiguro, "Development of composite powder for 3-dimensional facial appearance and evaluation of the optical properties," Fragrance Journal 39, 33-39 (2011).

11. T. M. Paschoalick, F. T. Garcia, P. J. A. Sobral, and A. M. Q. B. Habitante, "Characterization of some functional properties of edible films based on muscle proteins of Nile Tilapia," Hood Hydrocolloids 17, 419-427 (2003).

12. H. W. Jensen, S. R. Maeshner, M. Levoy, and P. Hanrahan, "A practical model for subsurface light transport," SIGGRAPH, ACM, 551-518 (2001).

13. R. R. Anderson, B. S John, and M. D. Parrish, "The optics of human skin," The Journal of Investigative Dermatology 77, 13-19 (1981).

14. R. Demirli, P. Otto, R. Viswanathan, S. Patwardhan, and J. Larkey, "RBX ${ }^{\mathrm{TM}}$ technology overview," Canfield Systems White Paper (2007).

15. A. Krishnaswamy and G. V. G. Baranoski, "A study on skin optics," Technical Report CS-2004-01, University of Waterloo, Waterloo, Ontario, Canada, January (2004).

16. L. Steve, J. C. Jacques, and K. L. Ramella-Roman, "Imaging skin pathology with polarized light," Journal of Biomedical Optics 7, 329-340 (2002).

17. Y. Masuda, "Methodology for evaluation of skin transparency and skin darkness," Fragrance 38, 37-43 (2010). 